\title{
Virtual glaucoma clinics: patient acceptance and quality of patient education compared to standard clinics
}

This article was published in the following Dove Press journal:

Clinical Ophthalmology

24 April 2015

Number of times this article has been viewed

\author{
Jennifer H Court' \\ Michael W Austin ${ }^{1,2}$ \\ 'Department of Ophthalmology, \\ Singleton Hospital, Swansea, Wales, \\ UK; ' ${ }^{2}$ Department of Ophthalmology, \\ Neath Port Talbot Hospital, Swansea, \\ Wales, UK
}

Purpose: Virtual glaucoma clinics allow rapid, reliable patient assessment but the service should be acceptable to patients and concordance with treatment needs to be maintained with adequate patient education. This study compares experiences and understanding of patients reviewed via the virtual clinic versus the standard clinic by way of an extended patient satisfaction questionnaire (PSQ).

Patients and methods: One hundred PSQs were given to consecutive patients attending glaucoma clinics in October 2013. All 135 patients reviewed via the virtual clinic from April 2013 until August 2013 were sent postal PSQs in September 2013. Data were obtained for demographics, understanding of glaucoma, their condition, satisfaction with their experience, and quality of information. Responses were analyzed in conjunction with the clinical records. Results: Eighty-five percent of clinic patients and $63 \%$ of virtual clinic patients responded to the PSQ. The mean satisfaction score was over $4.3 / 5$ in all areas surveyed. Virtual clinic patients' understanding of their condition was very good, with $95 \%$ correctly identifying their diagnosis as glaucoma, $83 \%$ as ocular hypertension and $78 \%$ as suspects. There was no evidence to support inferior knowledge or self-perceived understanding compared to standard clinic patients. Follow-up patients knew more about glaucoma than new patients. Over $95 \%$ of patients found our information leaflet useful. Forty percent of patients sought additional information but less than $20 \%$ used the internet for this.

Conclusion: A substantial proportion of glaucoma pathway patients may be seen by nonmedical staff supervised by glaucoma specialists via virtual clinics. Patients are accepting of this format, reporting high levels of satisfaction and non-inferior knowledge to those seen in standard clinics.

Keywords: glaucoma, care-pathway, satisfaction, questionnaire, education, service-delivery

\section{Introduction}

Virtual glaucoma clinics allow rapid and reliable patient assessment, ${ }^{1-4}$ with data collected by nursing and non-medical staff reviewed virtually by a senior ophthalmologist. However, with rapid throughput and loss of chair-time with an ophthalmologist it is important to confirm that patients remain satisfied with this aspect of service delivery and that patient education remains adequate to provide knowledge and awareness sufficient to maintain concordance with therapy and follow-up. The purpose of this study is to investigate non-inferiority of a new virtual clinic format by comparing experiences of patients managed via this pathway with a control group reviewed via the "standard" clinic, and to compare patients" understanding of their condition and management.
Correspondence: Jennifer H Court Department of Ophthalmology, Singleton Hospital, Sketty Lane, Swansea SA2 8QA, Wales

Tel +44 I792 285036

Fax +44 I792 285839

Email jennifer.court@wales.nhs.uk 


\section{Methods}

For several years we have operated a system of virtual clinic review. Clinical data obtained by an internally accredited ophthalmic nurse practitioner is reviewed virtually by a consultant ophthalmologist making the clinical decision. Data comprises of standardized history and slit lamp examination, intraocular pressure (IOP), optic disc imaging (Heidelberg Retina Tomograph [Heidelberg Engineering, Heidelberg, Germany], and/or Kowa Non-mydriatic 3D [Kowa Medical, Hamamatsu, Japan]) and Humphrey 24-2 visual fields (Carl Zeiss Meditec AG, Jena, Germany). This model of care is applicable to all newly referred patients and for follow-up patients for whom a virtual clinic review had previously been requested or who needed to have their next clinic appointment details specified eg, need for resetting of a previous date due to IOP not in target range, possible progression of visual field or optic disc parameters.

The clinical competencies of the non-medical staff regarding slit lamp examination of the eye, measurement of IOP, interpretation of visual fields, and clinical decision making had previously been confirmed as part of the institutional accreditation process. This is based on the same scheme of assessment as for medical ophthalmic trainees using the portfolio system of the Royal College of Ophthalmologists.

All 135 patients reviewed via the virtual clinic from April to August 2013 were identified prospectively for this study and recorded in a database. They were sent general and patient satisfaction questionnaires (modified 'QUality Of care Through the patient's Eyes' QUOTE questionnaire, Nijkamp et al $)^{5,6}$ by post in September 2013 . For the control group 100 patients attending standard glaucoma clinics in October 2013 were given questionnaires when they attended the clinic. The patients were selected on the basis of being consecutive attenders at the "actual" clinic and consecutive patients presented via their clinic notes to the consultant (MA) undertaking the virtual clinic review.

Data were obtained for demographics, understanding of glaucoma, their own condition and its management, together with their satisfaction with their experience (modified QUOTE questionnaire), ${ }^{5}$ and quality of information received. Patient responses were analyzed in conjunction with clinical data obtained from the clinical records.

\section{Results}

The demographics of the standard and virtual clinic patients (Table 1) were comparable other than the proportion of new to follow-up patients $(0.14$ versus 0.34 , respectively, $P<0.01)$. The questionnaire results were analyzed by looking at the analysis of variance (ANOVA) using SPSS Statistics (IBM Corporation, Armonk, NY, US).
Table I Patient demographics

\begin{tabular}{lll}
\hline & Standard clinic & Virtual clinic \\
\hline Number of questionnaires & 100 & 135 \\
Number of responses & 85 & 85 \\
Response rate (\%) & 85 & 63 \\
Mean age (years) & 70 & 64 \\
Standard deviation age (years) & 11.8 & 13.4 \\
Age range (years) & $32-90$ & $30-93$ \\
Male:female ratio (\%) & $49: 51$ & $50: 50$ \\
New:follow-up ratio (\%) & $14: 86$ & $35: 64$ \\
\hline
\end{tabular}

Patients scored their satisfaction from 1 (very dissatisfied) to 5 (very satisfied) for a series of questions. The modal score for all questions in both groups was 5 out of 5 . The overall mean satisfaction score for all questions combined was 4.6 and 4.5 for the standard clinic and virtual clinic respectively and there was no evidence to suggest that patients reviewed via the virtual clinic felt disadvantaged compared to those consulting with a doctor face to face.

Patients were asked questions about their diagnosis and treatment and their answers were compared with the doctors' recorded diagnosis and treatment to assess individuals' understanding of their own condition (Figure 1). Understanding was high in the virtual clinic group, with $95 \%$ of patients correctly identifying their diagnosis as glaucoma, $83 \%$ as ocular hypertension, and $78 \%$ as glaucoma suspects. This is particularly reassuring when considering one third of these were new patients and had not consulted face to face with a doctor. No inferiority was demonstrated in knowledge between virtual and standard clinic patients despite this higher proportion of new patients in the virtual clinic group for whom there may be increased diagnostic uncertainty and fewer opportunities to receive information and ask questions.

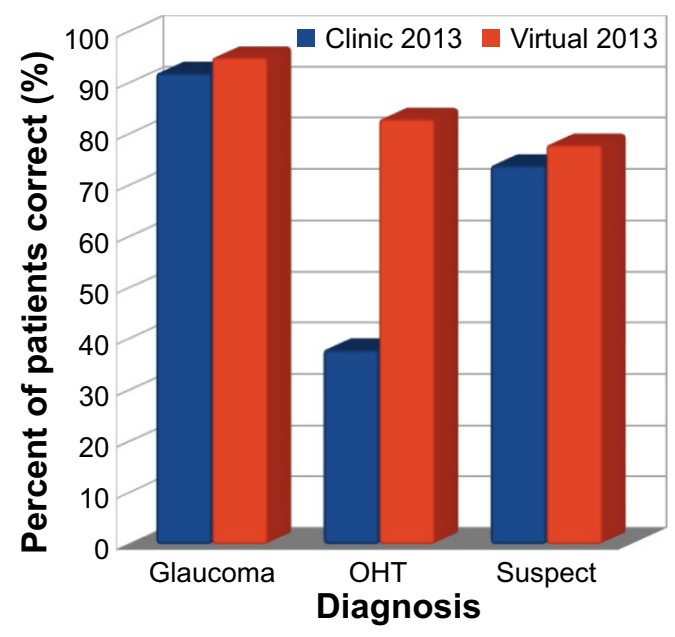

Figure I Patients' knowledge of their diagnosis. Abbreviation: $\mathrm{OHT}$, ocular hypertension. 
The study identified nine patients who had been started on treatment via the virtual clinic. This new treatment is issued via a treatment recommendation form sent to the patient to take to their general practitioner to alter their prescribing record. They are informed about this process at their initial appointment when their data are collected, receive a telephone call by the glaucoma nurse practitioner to discuss it, and are brought back to clinic for early review. Three patients were identified as requiring trabeculectomy surgery and attended the clinic to confirm and discuss this.

Patients' knowledge about glaucoma and self-perceived understanding were analyzed comparing the responses to each question for new versus follow up patients, whether they had received an information leaflet or not, and whether they had received a clinic report or not using ANOVA. Patients were asked to grade their understanding of glaucoma, their personal diagnosis, and their treatment (what it does and how to use it) from 5 (strongly agree) to 1 (strongly disagree) to gauge their confidence in their knowledge. Self-perceived understanding was high across the groups with modal scores of 4 out of 5 for understanding of glaucoma and their diagnosis for both the standard and virtual clinic groups, and 5 out of 5 for understanding of their treatment and no evidence was found of inferiority $(P>0.05)$. The use of written information is proposed as a key factor in improving patient understanding and information retention, and virtual clinic patients who received a leaflet felt they understood what their drops did better than those who did not $(P=0.04)$. There was insufficient evidence to support a difference between new and follow-up patients or those who had received a clinic report or not.

Patients answered seven "did you know?" questions in a simple "yes" or "no" format to assess their knowledge about glaucoma in general (Figure 2). Follow-up patients reviewed in the virtual clinic knew more about glaucoma than new patients $(P=0.02)$ reflecting the importance of repeating information to improve understanding and retention of information. The increased proportion of follow-up to new patients in the standard clinic versus the virtual clinic was thus a confounding factor in the comparison between the clinic and virtual groups but overall there was no evidence of a significant difference in knowledge. There was insufficient evidence to support a difference between those who had received an information leaflet or not or those who had received a clinic report or not.

It is protocol for all patients to be given our information leaflet called "So, Have I Got Glaucoma?" This leaflet was written by the lead glaucoma consultant (MA) and approved for patient-suitable language and use by the institution's editorial board. However, two thirds of standard clinic patients and one third of virtual clinic patients did not recall receiving one (in some cases contrary to documented issuing). Six percent of patients admitted getting but not reading the leaflet. There is therefore some scope for improvement in the giving of written information and efforts are made to keep the clinics well stocked with leaflets and give regular reminders to medical and non-medical staff to give them to patients. Over $95 \%$ of patients who read the leaflet understood it and found it helpful. New patients and patients seen by the nurse practitioners were more likely to have received a leaflet. There was insufficient evidence in this study to support the hypothesis that those who received information leaflets had better knowledge than those who did not, though there was evidence that patients did feel they understood what their treatment did better if they had received a leaflet as discussed above.

\section{Question key:}

1. Did you know that glaucoma is a condition that affects the optic nerve that connects the eye to the brain?

2. Did you know that glaucoma is usually caused by high pressure in the eye?

3. Did you know that some people have glaucoma without having high pressure in the eye?

4. Did you know that it is possible to have high pressure in the eye without getting glaucoma (this is called ocular hypertension)?

5. Did you know that for some people it is difficult to decide whether they have glaucoma or not (these people are called "glaucoma suspects")?

6. Did you know that with treatment most people with glaucoma will keep good eyesight?

7. Did you know that glaucoma is the second commonest cause of severe visual impairment (blindness) in Wales?

Figure 2 Patients' knowledge about glaucoma. 
Forty percent of standard clinic and $40 \%$ of virtual clinic patients said they had sought further information about glaucoma. Approximately one third of these in both groups had not received our information leaflet.

Fifty-six percent of patients had personal access to the internet and $28 \%$ had access via a relative or friend. However, only $26 \%$ of those with personal access had searched for information about glaucoma online. All but two patients understood the information they found and felt it was helpful. The International Glaucoma Association website is recommended in our information leaflet and clinic outcome report but few patients had visited it (Figure 3).

Virtual clinic patients are sent a standard written report from the consultant following the virtual review of their results. Eighty-seven percent of patients felt outcome reports were a good idea. Clinic patients were given a sample report to read as part of the questionnaire. Ninetyfive percent of virtual and clinic patients who received and read the report said they understood it and $88 \%$ felt it was helpful. One percent of virtual clinic patients and 17\% of standard clinic patients said they would object to receiving a prescription for a new eye drop through the post. Patients are telephoned by the nurse practitioner to initiate a new treatment in addition to the written report and prescription as a result.

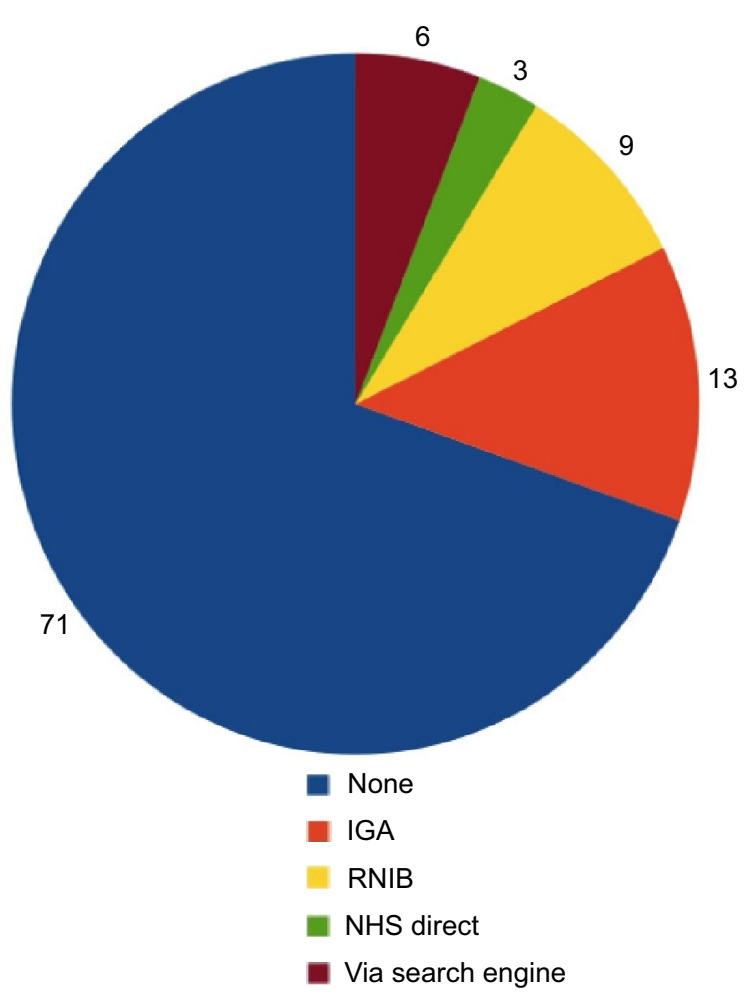

Figure 3 Websites visited for additional information (\% of patients).

Abbreviations: IGA, International Glaucoma Association; RNIB, Royal National Institute of Blind People; NHS, National Health Service.
There were no statistically significant differences for any parameters on account of age.

\section{Discussion}

Patients expressed high levels of satisfaction with our glaucoma service. Satisfaction was high regardless of whether they were reviewed via the virtual or standard clinic.

Patients' understanding of their diagnosis and treatment was high across both groups and self-perceived understanding of glaucoma, their own condition, and treatment is high. The majority of patients know that glaucoma affects the optic nerve, is often caused by high IOP and with treatment vision usually remains good. The nuances of normal tension glaucoma, ocular-hypertension and glaucoma suspects were less well understood but felt to be less important to patients and their likely concordance with management. The acceptance of a model of care, whilst important from the point of view of patient experience, cannot of itself guarantee any particular level of concordance with care or adherence to treatment. We would suggest though that it would be counterintuitive to suggest the contrary.

No evidence was found to support a hypothesis that patients reviewed virtually rather than in person by a doctor feel they receive an inferior quality service. It would be useful to know the converse, that patients seen in person would feel equally well supported if they had the ophthalmologist input to their care via a virtual clinic evaluation. A Spanish study by Blázquez et $\mathrm{al}^{7}$ reported high levels of patient satisfaction with a telemedicine glaucoma screening program among an at-risk population but this is the first study we are aware of that looks at the satisfaction of patients reviewed virtually who have already been referred into the hospital eye care setting compared to the traditional standard clinic format.

The similar levels of satisfaction and understanding between the standard and virtual clinic patients despite the significantly higher proportion of new patients in the virtual clinic group suggests that this model of care is acceptable for both new and follow-up patients.

The study groups did not include sufficient numbers of patients with advanced glaucoma as to be able to allow for specific conclusions to be drawn as to the more complex therapeutic decision making and therefore communication requirements for such patients. Nevertheless, the additional clinic capacity gained by use of virtual clinic working for less demanding individuals would allow more time for those with greater needs. The need for IOP lowering surgery is invariably based on the levels of recorded IOP compared with "target" at maximum tolerated medical therapy and the progression of any visual field defect. The tolerability 
of medical therapy is able to be assessed by a non-medical practitioner. IOP (by an accredited practitioner) and visual fields data (with progression software) were available in the virtual clinic data. It is of course important to regularly confirm the diagnostic, management, and communications competencies of all clinical staff, both medical and from professions allied to medicine via schemes for continuing education and appraisal to maintain a high quality service.

Patients value written information in the form of information leaflets and clinic reports and work needs to be done to improve the proportion who receive these; they remain the main source of additional information for patients. All patients attending the glaucoma clinic, whether they have been seen by a member of the medical staff or a practitioner from a profession allied to medicine and whether or not their clinical data are scheduled for virtual clinic review have access to additional support and information from the on-site eye clinic liaison officer. Our future work will address the contributions made to satisfaction and awareness by combinations of ophthalmologist, nurse, and eye clinic liaison officer input.

Virtual clinic models help optimize the use of resources to help address the large over-demand on hospital eye services. A recent large retrospective study by Wright and Diamond ${ }^{4}$ highlighted this need and showed the benefits to safety and efficiency from having specialist supervision of decisions made by optometrists about glaucoma patients' care. Our model utilizes non-ophthalmologists for data collection and education of patients but not in the decision making. Further studies looking at cost-benefit analysis and optimizing the care pathway are needed to find the overall best model of care.

Whilst our study supports the comparability of virtual clinic enhancement of non-medical practitioner clinics with standard clinics with respect to understanding of their condition and overall experience, there remains considerable room for improvement in significant numbers of patients in each study group.

\section{Conclusion}

The virtual clinic model allows a substantial proportion of glaucoma pathway patients to be seen by non-medical staff and yet be supervised by a glaucoma specialist. This allows rapid throughput to address the increasing demands on our service and allows doctors' time to be prioritized to the most complex patients and those at greatest risk of progression. The results of this survey shows that patients seen in this way receive adequate information and education about their condition, which we feel is important to help maintain appreciation of the value of attending follow-up appointments and concordance with management. Patients are accepting of this type of care and report high levels of satisfaction with the nurse-led clinic experience.

\section{Acknowledgments}

The authors would like to acknowledge the hardworking and conscientious staff at Singleton and Neath Port Talbot Eye clinics, in particular Fiona Thomas and the glaucoma nurse practitioners. The authors would like to acknowledge Dr S Zhou, Swansea University, for his assistance with the SPSS analysis of the data.

\section{Disclosure}

The authors report no conflicts of interest in this work.

\section{References}

1. Rathod D, Win T, Pickering S, Austin M. Incorporation of a virtual assessment into a care pathway for initial glaucoma management: feasibility study. Clin Exp Ophthalmol. 2008;36(6):543-546.

2. Trikha S, Macgregor C, Jeffery M, Kirwan J. The Portsmouth-based refinement scheme: a role for virtual clinics in the future? Eye (Lond). 2012;26(10):1288-1294.

3. Syam P, Rughani K, Vardy SJ, et al. The Peterborough scheme for community specialist optometrists in glaucoma: a feasibility study. Eye (Lond). 2010;24(7):1156-1164.

4. Wright HR, Diamond JP. Service innovation in glaucoma management: using a web-based electronic patient record to facilitate virtual specialist supervision of a shared care glaucoma programme. $\mathrm{Br} J$ Ophthalmol. 2015;99(3):313-317.

5. Nijkamp MD, Sixma HJ, Afman H, et al. Quality of care from the perspective of the cataract patient: the reliability and validity of the QUOTE-Cataract. Br J Ophthalmol. 2002;86(8):840-842.

6. van Campen C, Sixma H, Friele RD, Kerssens JJ, Peters L. Quality of care and patient satisfaction: a review of measuring instruments. Med Care Res Rev. 1995;52(1):109-133.

7. Blázquez F, Sebastián MA, Antón A. Detección de glaucoma mediante telemedicina con SisGlaTel: satisfacción y aceptación entre los participantes y problemas detectados [Detection of glaucoma using SisGlaTel: acceptability and satisfaction among participants, and problems detected] Arch Soc Esp Oftalmol. 2008;83(9):533-538. Spanish.
Clinical Ophthalmology

\section{Publish your work in this journal}

Clinical Ophthalmology is an international, peer-reviewed journal covering all subspecialties within ophthalmology. Key topics include: Optometry; Visual science; Pharmacology and drug therapy in eye diseases; Basic Sciences; Primary and Secondary eye care; Patient Safety and Quality of Care Improvements. This journal is indexed on

Submit your manuscript here: http://www.dovepress.com/clinical-ophthalmology-journal

\section{Dovepress}

PubMed Central and CAS, and is the official journal of The Society of Clinical Ophthalmology (SCO). The manuscript management system is completely online and includes a very quick and fair peer-review system, which is all easy to use. Visit http://www.dovepress.com/ testimonials.php to read real quotes from published authors. 